\title{
Following the written path - a patient's journey to finding care!
}

\author{
samhati Mondal ${ }^{1}$ and Lloyd Allen ${ }^{1}$ \\ ${ }^{1}$ University of Maryland School of Medicine
}

April 23, 2021

\begin{abstract}
Incidental cardiac tumors are rare and mostly detected on autopsy as patients largely remain asymptomatic. However, diagnosis of an incidental cardiac mass on unrelated work up can pose significant ethical and clinical challenge to the care team. Surgical resection has been the most successful intervention for most primary cardiac tumors however, it involves cardiopulmonary bypass assisted major surgery which is not risk free either. Cardiac lipoma is the second most common primary cardiac benign tumor. We report a case of a young otherwise healthy patient who reported have a cardiac lipoma on computerized tomography (CT ) scan that was done to rule out kidney stone.
\end{abstract}

Following the written path - a patient's journey to finding care!

Llyod Barrera Allen, MD; ${ }^{1}$ Samhati Mondal, MBBS, MD ${ }^{1}$

Affiliations -

Department of Anesthesiology, University of Maryland School of Medicine, Baltimore, MD

Corresponding Author

Samhati Mondal, MBBS, MD

Assistant Professor, Department of Anesthesiology

University of Maryland School of Medicine

22 S Greene Street

Baltimore, MD, 21201

Tel: $410-328-9813$

Fax: 410-328-3092

Manuscript Word Count: 650 Figure -2 Videos- 4

Conflicts of Interest - None of the authors have any conflicts of interest

Author contributions:

LBA : Searching literature, drafting of the manuscript, revising the draft for critically important intellectual content

SM: Conceptualization, design, obtaining images and videos, drafting of manuscript and revising for critically important intellectual content.

All authors approve the final submitted version and agree to be accountable for all aspects of the work presented. 
Following the written path - a patient's journey to finding care!

Dear Editor,

Incidental cardiac tumor is mostly diagnosed on autopsies as majority of the patients remain asymptomatic during their lifetime.[1] But when an incidental primary cardiac tumor is diagnosed, it not only poses serious medical as well as ethical challenges to care team, an otherwise asymptomatic patient himself may become surprised and rather baffled with such an unexpected yet critical diagnosis. Information technology (IT) and internet connectivity have made significant advancement in our society over last two decades. Modern medicine has benefitted by the advancement in IT and internet connectivity too. I was very surprised to receive an email from 32 year old cardio healthy gentleman that he has been recently given a diagnosis of an incidental cardiac tumor that was caught by a computerized tomography (CT) scan done for kidney stone work up. Given the rarity of the situation and his asymptomatic status, further work up including magnetic resonant imaging (MRI) and transthoracic echocardiography (TTE) were performed to confirm the diagnosis. When all these imaging were suggestive of a cardiac tumor despite not being able to conclusively delineate the extent and nature of the tumor, this young, tech-savvy patient started reading about primary cardiac tumor. That is when he came across my previous article 'Near Total Occlusion of Right Ventricle by Cardiac Mass' which was published at Journal of Cardiothoracic and Vascular Anesthesia (JCVA) and knowing that we both are geographically from the same state (MD), he emailed me if I can connect him with some cardiothoracic (CT) surgery experts (Figure 1). [2]

I was very surprised to receive that request however, I suggested him few of the renowned CT surgeons who are well known for cardiac tumor surgery at my institutions. Few weeks later, he was scheduled for surgical resection of the cardiac tumor with one of the surgeons, I recommended and oddly enough, I was assigned to be his CT anesthesiologist.

Upon induction of an uneventful general endotracheal anesthesia and placement of lines, a transesophageal echocardiography (TEE) was performed. TEE showed a well-defined left ventricular mass abutting the lateral wall measuring approximately $3 \mathrm{~cm}$ by $2 \mathrm{~cm}$. (Figure 2; Video 1 ). There was concern for antero-lateral (AL) papillary muscle as well mitral valve apparatus involvement, but TEE was able to demonstrate that most of cordal attachment to AL papillary muscle was not invaded by the tumor and intact (Video 2). TEE also showed normal biventricular function along with absence of mitral regurgitation (MR) (Video 3). After establishment of cardiopulmonary bypass (CPB), he underwent ventriculotomy lateral to apex of the left ventricle, and he was found to have a soft yellow mass at the base of the anterolateral (AL) papillary muscle with no clear plane between mass and muscle. The mass was resected, and it appeared to not significantly affect the papillary muscle or mitral valve (MV) apparatus. He had an uneventful weaning off from CBP. His post CPB TEE showed normal biventricular function and no significant mitral regurgitation. (Video 4). Histopathology examination of the resected mass has been reported to a lipoma, mixed with some hamartomatous component (benign).

Primary cardiac tumor is very rare and most of the incidental cardiac tumor is diagnosed on autopsy.[1] However, when an incidental cardiac tumor is diagnosed, it can be challenging decision for patient as well as care team, especially surgical resection of the same involves a major surgery including assistance of cardiopulmonary bypass and sternotomy. Cardiac lipoma is a benign primary cardiac tumor and it is second most common in that category following myxoma.[3] Lipoma can be of epicardial, pericardial or subendocardial origin. [4] Symptomatology depends on location and size of the lipoma. [5]

In this case, an educated, asymptomatic, young but baffled patient by reviewing literature reached out to author/anesthesiologist of an article on cardiac mass. While I am glad to be able to help the patient out, fair share of credit goes to advancement of IT and easy internet access.

\section{Legends -}

Figure -

Figure 1 - Email of the patient showing he reached out to CT anesthesiologist 


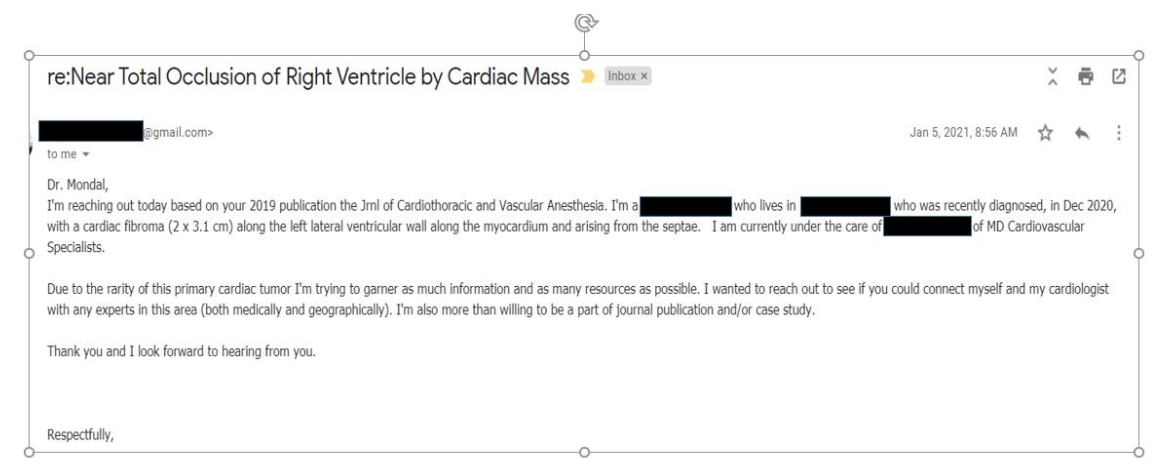

Figure 2 - 3D TEE showing cardiac mass $3 \mathrm{x} 2 \mathrm{~cm}$

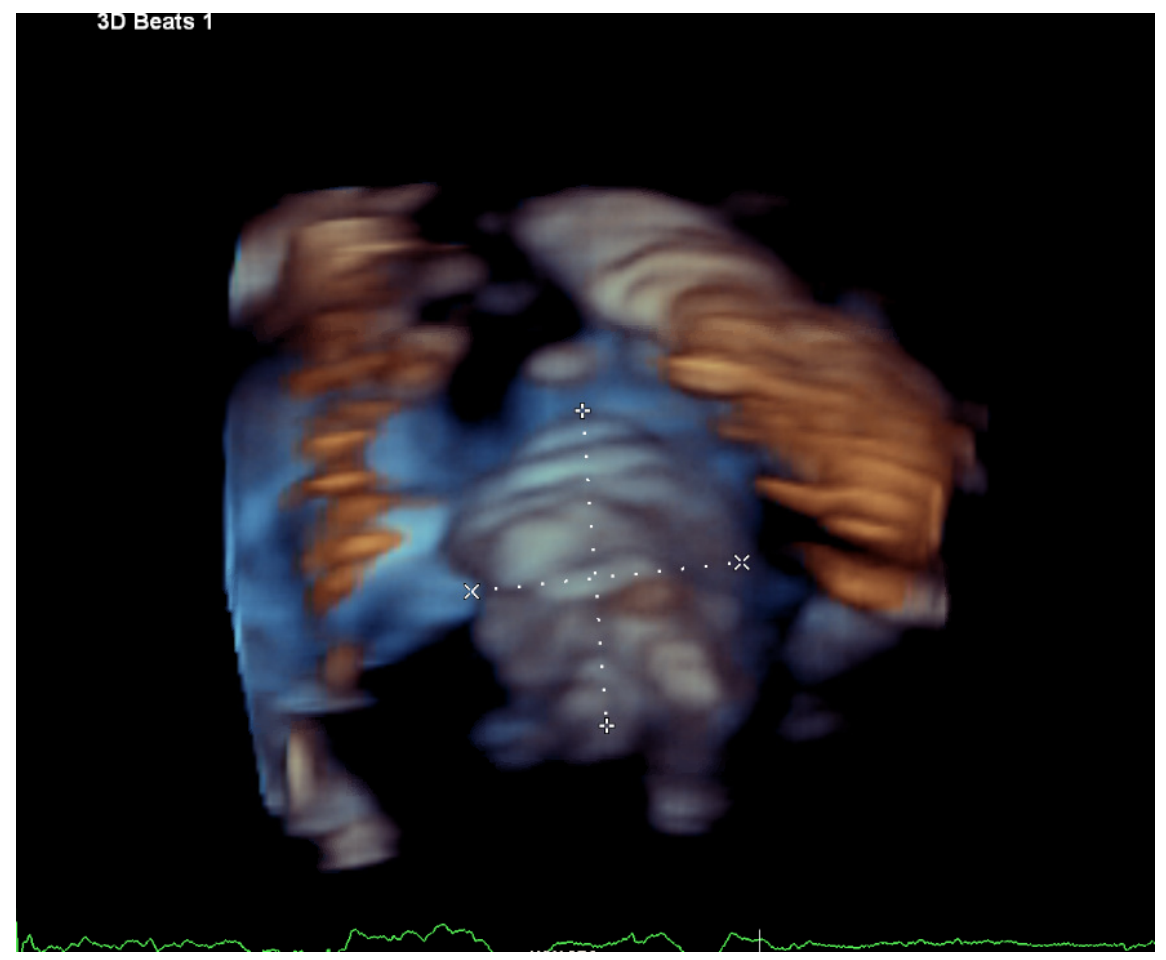

\section{Video -}

Video 1 - 3D TEE image showing LV mass without affecting mitral valve

Video 2 - Transgastric midpapillary view concerning involvement of AL papillary muscle on short axis but intact cords visible on long axis view

Video 3 - TEE ME long axis view showing absence of mitral regurgitation (MR)

Video 4- post operative TEE: ME 4 chamber view showing absence of cardiac mass and MR

References

1. Staudt, G.E., et al., Incidental Discovery of an Atypical Cardiac Tumor. J Cardiovasc Echogr, 2018. 28 (3): p. 198-200. 
2. Mondal, S., J. Jubar, and M.P. Kostibas, Near Total Occlusion of Right Ventricle by Cardiac Mass. J Cardiothorac Vasc Anesth, 2019.33 (7): p. 2085-2090.

3. Barbuto, L., et al., Humongous right atrial lipoma: a correlative CT and MR case report. Quant Imaging Med Surg, 2015.5 (5): p. 774-7.

4. Liu, L., et al., Echocardiographical findings of giant cardiac lipoma: A case report. Medicine (Baltimore), 2019. 98 (8): p. e14456.

5. D'Souza, J., et al., Invasive Cardiac Lipoma: a case report and review of literature. BMC Cardiovasc Disord, 2017. 17 (1): p. 28.

\section{Hosted file}

Figure_Cardiac lipoma_0415.pdf available at https://authorea.com/users/409644/articles/ 519231-following-the-written-path-a-patient-s-journey-to-finding-care 